\title{
Status of the Baikal-GVD Neutrino Telescope
}

\author{
A.D. Avrorin ${ }^{1}, A . V$. Avrorin $^{1}$, V.M. Aynutdinov ${ }^{1, *}, R$. Bannash $^{7}, I . A$. Belolaptikov $^{2}$, \\ V.B. Brudanin ${ }^{2}$, N.M. Budnev ${ }^{3}, A . A$. Doroshenko ${ }^{1}$, G.V. Domogatsky ${ }^{1}, R$. Dvornický $\dot{y}^{2 ;}$,

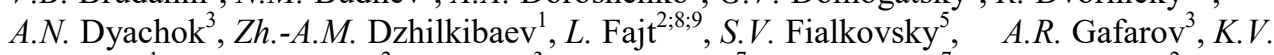 \\ Golubkov $^{1}$, N.S. Gorshkov ${ }^{2}$, T.I. Gres ${ }^{3}$, K.G. $\mathrm{Kebkal}^{7}$, O.G. Kebkal ${ }^{7}$, E.V. Khramov ${ }^{2}$, M.M. \\ Kolbin $^{2}, K . V$. Konischev $^{2}$, A.V. Korobchenko ${ }^{2}$, A.P. Koshechkin ${ }^{1}, V . A . \mathrm{Kozhin}^{4}$, M.V.

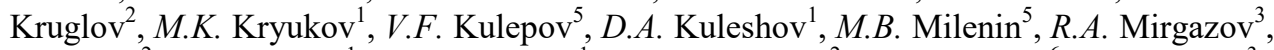 \\ V. Nazari ${ }^{2}$, A.I. Panfilov ${ }^{1}$, D.P. Petukhov ${ }^{1}$, E.N. Pliskovsky ${ }^{2}$, M.I. Rozanov ${ }^{6}$, E.V. Rjabov ${ }^{3}$, \\ V.D. Rushay ${ }^{2}$, G.B. Safronov ${ }^{2}, F$. Simkovic ${ }^{2 ; 8}, A . V$. Skurichin ${ }^{4}$, B.A. Shaybonov ${ }^{2}, A . G$. \\ Solovjev $^{2}$, M.N. Sorokovikov ${ }^{2}, M . D$. Shelepov ${ }^{1}, O . V$. Suvorova ${ }^{1}, \quad I$. Shtekl $^{2 ; 9}$, V.A. \\ Tabolenko $^{3}, B . A$. Tarashansky ${ }^{3}, S . A$. Yakovlev $^{7}$, and $A . V$. Zagorodnikov $^{3}$ \\ ${ }^{1}$ Institute for Nuclear Research, Moscow, 117312 Russia \\ ${ }^{2}$ Joint Institute for Nuclear Research, Dubna, 141980 Russia \\ ${ }^{3}$ Irkutsk State University, Irkutsk, 664003 Russia \\ ${ }^{4}$ Institute of Nuclear Physics, Moscow State University, Moscow, 119991 Russia \\ ${ }^{5}$ Nizhni Novgorod State Technical University, Nizhni Novgorod, 603950 Russia \\ ${ }^{6}$ St. Petersburg State Marine Technical University, St. Petersburg, 190008 Russia \\ ${ }^{7}$ EvoLogics, 13355, Berlin, Germany \\ ${ }^{8}$ Comenius University, 81499, Bratislava, Slovakia \\ ${ }^{9}$ Czech Technical University, 16000, Prague, Czech Republic
}

\begin{abstract}
Currently in Lake Baikal a new-generation neutrino telescope is being deployed: Baikal-GVD, a deep underwater Cherenkov detector on the cubic-kilometer scale. This paper presents the status of the detector implementation and the first physical results obtained with the existing configuration.
\end{abstract}

\section{Introduction}

Deep-sea neutrino telescopes are designed to study high-energy processes in astrophysical objects and in the Universe as a whole using high-energy neutrinos. Neutrino telescopes of the first generation were created sequentially in Lake Baikal (NT200, 1998), in Antarctica at the South pole (AMANDA, 2000) and in the Mediterranean Sea (ANTARES, 2008). The successful operation of these detectors initiated the creation of the next generation of neutrino telescopes, with a scale of a cubic kilometre. IceCube, Baikal-GVD and KM3NeT are the most relevant of such neutrino telescopes in operation or currently being constructed. This article is devoted to the creation of a large-scale neutrino telescope in Lake Baikal. The plan for the Baikal-GVD telescope includes two stages. As a result of the completion of the first stage $(2020-2021)$, a facility consisting of 2304 optical modules

* Corresponding author: aynutdin@yandex.ru 
with an effective volume of $0.4 \mathrm{~km}^{3}$ will be created. At the next stage, the effective volume will be increased to $1.5 \mathrm{~km}^{3}$.

Initially the Baikal-GVD collaboration included only Russian research institutions: from Moscow, Dubna, Irkutsk, Nizhni Novgorod and St. Petersburg. Meanwhile the nine partners include also the Universities of Prague and Bratislava and the EvoLogics company in Berlin which develops the advanced acoustic positioning system.

\section{The BAIKAL-GVD neutrino telescope}

The Baikal-GVD neutrino telescope $[1,2]$ is a spatial structure of optical modules (OMs) grouped into clusters. Each cluster comprises $288 \mathrm{OMs}$, placed on 8 vertical strings. The string length is about $525 \mathrm{~m}$, the distance between the strings $60 \mathrm{~m}$, the distance between the clusters $300 \mathrm{~m}$. Work on the detector implementation started in 2009. The final design of Baikal-GVD was completed in 2013, followed by the phased deployment of the detector.

By 2018, three clusters were commissioned. An artistic view of the installation is shown in Fig. 1. The right part of the figure shows the cumulative number of events detected between April 19 and August 21, 2018. Event rates are approximately the same for all clusters and range from 40 to $90 \mathrm{~Hz}$. depending on the intensity of the background luminosity of the water.
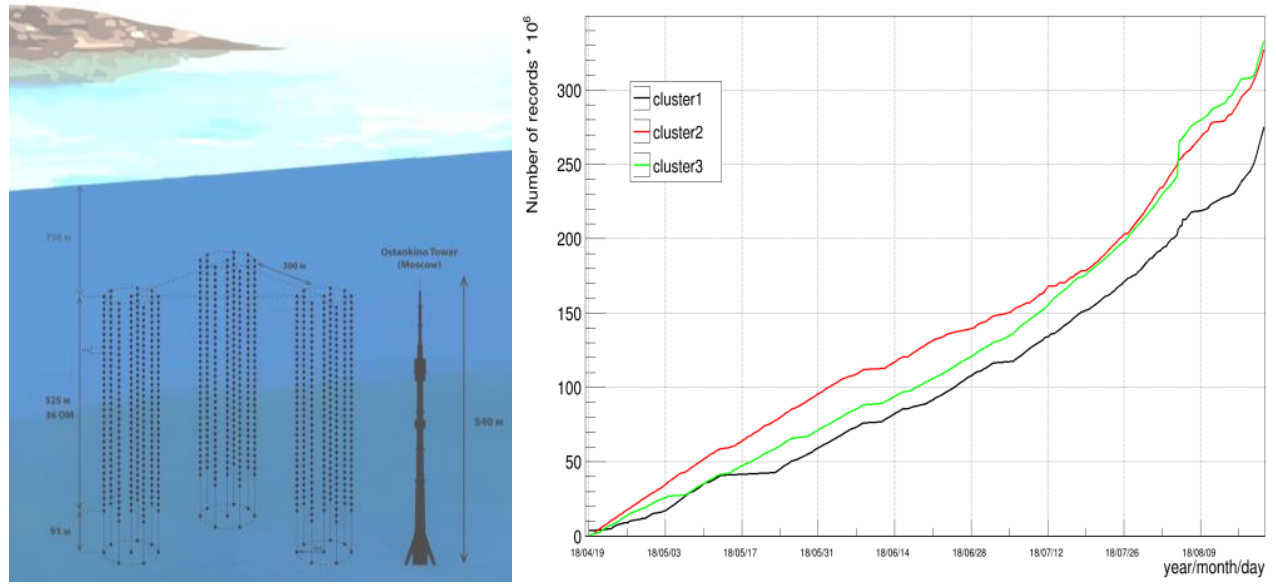

Figure 1. Artistic view of Baikal-GVD in 2018 (left) and cumulative number of the events detected with three clusters (right).

The configuration and technical implementation of the telescope is largely determined by the way it is deployed. Deployment is carried out from the ice cover of the lake. The deep-sea modules are installed on after the other along the load-carrying cable and submerged under water by means of winches (see Fig. 2, top). After installation of each optical module it is tested for proper functioning. After installation of each string section (12 OMs) and of the string as a whole, further test procedures are performed. During testing, OMs are switched to a test-pulse mode.

Strings are assembled by groups of three persons per string. The time for installing one string including all test procedures is 3-4 days, depending on weather conditions. At the last stage of cluster assembly, all strings are connected to the cluster DAQ center that is located at a depth of only 30 meters. The cluster center is connected to the shore center via 
a hybrid opto-electrical cable, which is deployed with the help of ice-cutting equipment specially designed for the Baikal project (Fig. 2, bottom).
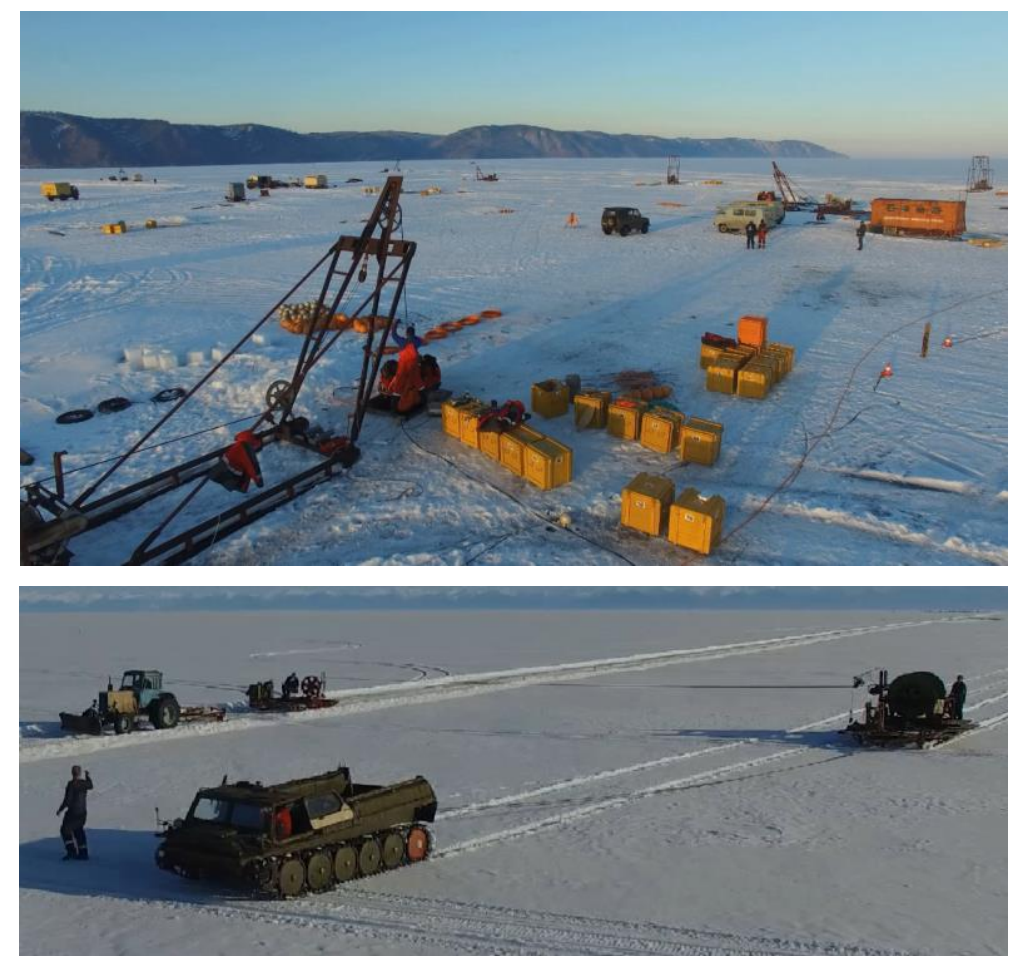

Figure 2. Ice camp (top) and cable laying equipment (bottom).

The principle of operation of the detector is based on the registration of Cherenkov radiation of secondary muons and cascade showers produced in neutrino interactions. The light-sensitive elements of the OMs are PMTs of the type R7081-100 with a hemispherical photocathode diameter of 10 inches and a quantum sensitivity of $\sim 35 \%$ [3]. The PMT signals are amplified and transmitted to the ADC units to measure the pulse shape with a sampling rate of $200 \mathrm{MHz}$ [4]. Each ADC unit is equipped with quartz oscillators that allow for measuring the time of the event registration. A global trigger signal is used to unify the event times measured by different ADC units within the same cluster to a single time scale with accuracy $\sim 2 \mathrm{~ns}$. The trigger condition is a coincidence of pulses from any neighbouring optical modules with thresholds of 1.5 and 4 photoelectrons. The cluster trigger rate for these conditions does not exceed $100 \mathrm{~Hz}$.

ADC units are located in separate deep-sea modules that serve 12 OMs (a section of OMs). Pulse shape analysis allows for determining the number of signals coming from the PMT, their charges and registration times. On the basis of measurements of the time of registration of the pulses and their charges on all triggered channels, the main parameters of an event are determined: the direction and energy of muons and cascade showers, respectively. The accuracy of the direction reconstruction of muon tracks amounts to $0.5^{\circ}$, and that of the energy of cascade showers to about $20 \%[5,6]$.

For amplitude and time calibration of the detector, LED calibration sources are used. They are located both in the optical modules and in separate deep-water glass spheres (LED matrix). Each OM comprises two LEDs, the light from which spreads up along the string. These LEDs are employed for channel calibration within sections of OMs. The LED matrices are used for inter-section time calibration. Each matrix comprises 12 synchronized 
LEDs flashers, from which pulses can be detected by several sections. The accuracy of the time calibration is about $2 \mathrm{~ns}$ [7].

Laser sources are used for inter-cluster calibration. The laser calibration source generates light flashes with about $10^{15}$ photons at maximum power running. The laser wavelength is $532 \mathrm{~nm}$, the pulse duration about $1 \mathrm{~ns}$. The laser is located approximately in the center of the group of three clusters, at the level of the middle of the lower sections of the optical modules (see Figure 3). The laser is equipped with a diffuser that forms a quasiisotropic light flux. One laser source allows calibrating three clusters simultaneously.

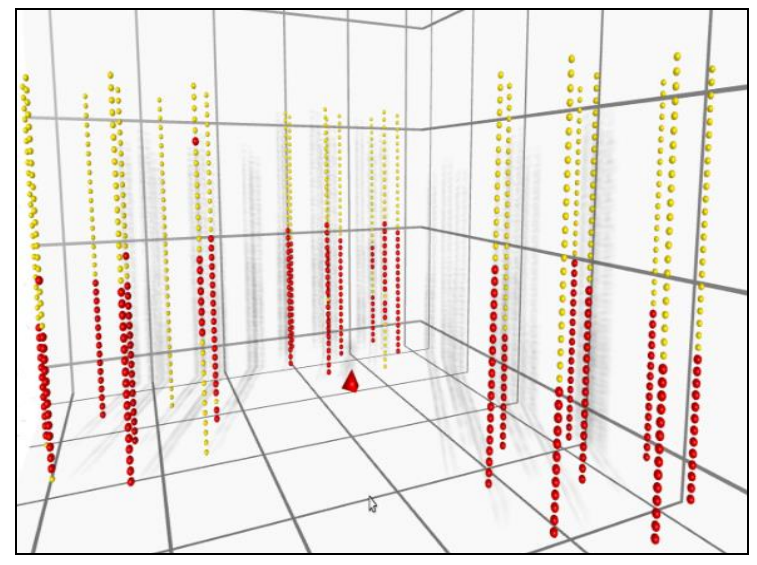

Figure 3. An example of a laser flash detected by three GVD clusters. The position of the laser is indicated by a triangle, red circles mark the triggered channels.

To synchronize the operation of different clusters, the arrival time of the global trigger is measured in each of them. For these measurements a common clock source is used, the signals from which are transmitted via fiber-optic lines to all clusters of the telescope. Reference to the World time is carried out using GPS/GLONASS and a precision server GMR-5000 with a built-in rubidium oscillator: time reference accuracy $\sim 15$ ns. The accuracy of inter-cluster synchronization was measured with the laser calibration source. Figure 4 shows the distribution of differences between times of triggers measured on two clusters.

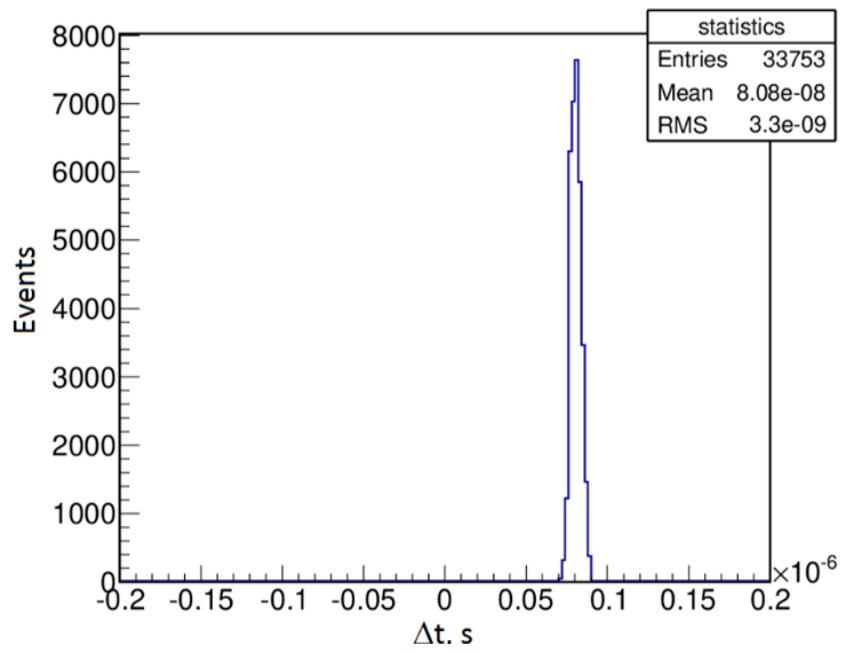

Figure 4. Time difference $\Delta t$ between global triggers of two clusters. 
The RMS of this distribution $(\sim 3 \mathrm{~ns})$ characterizes synchronization accuracy. The mean value $(81 \mathrm{~ns})$ correspond to the difference in the distance from the laser to the nearest strings of the clusters (121.3 m and $102.7 \mathrm{~m}$, respectively) which was measured with the help of the acoustic positioning system.

The coordinates of the optical modules are measured with an acoustic positioning system [8]. Four acoustic modems (AM) are installed on each string. The distances between neighboring AMs are about 180 meters along the string. The accuracy of the measurement of the AM coordinates is better than $5 \mathrm{~cm}$. The accuracy of determining the coordinates of the optical modules located between AMs is determined mainly by the uncertainty in the form of bending of the string. To estimate this uncertainty, an additional AM was installed in the center of the upper section of the string, in the area of the expected maximum bend. The difference between the measured AM coordinates and the results of their interpolation using the neighboring AMs is shown in Figure 5. The mean XY error is about $13 \mathrm{~cm}$, that corresponds to the accuracy of the registration time by the OM of less than $1 \mathrm{~ns}$.

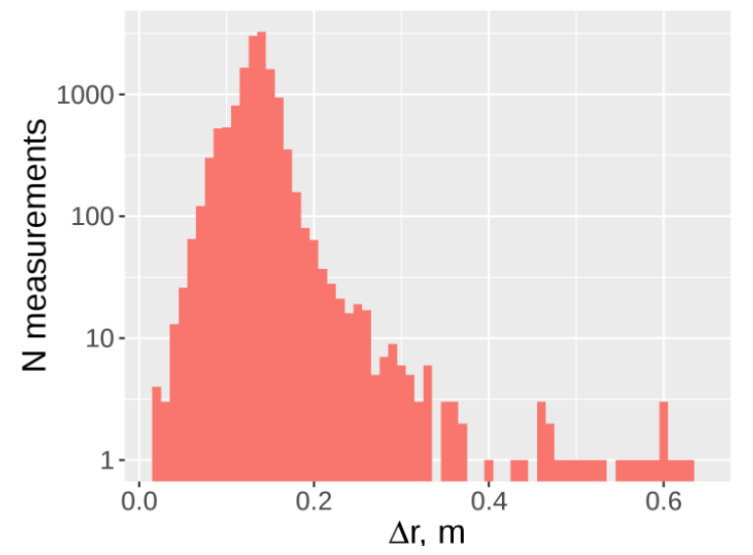

Figure 5. Distribution of differences between measured coordinates of the AM located in the centre of the top section and the interpolated one (April $\div$ September 2018).

\section{First results}

The modular structure of the registration system of Baikal-GVD allows performing physics studies already in early stages of construction. The neutrino telescope has been in permanent operation since April 2015, when the first GVD cluster was commissioned. The priority tasks were to study muon neutrinos and high-energy cascades to search for astrophysical neutrino sources, and to search for neutrino events correlating in time and direction with sources of variable luminosity. Muon neutrinos were selected by detection of muons coming from the lower hemisphere of the Earth. Each GVD cluster detects about one event of this type per day (depending on the trigger conditions). As a result of the search for the neutrinos detected via the cascade mode, two events were selected as candidates for astrophysical neutrinos [9, 10]. The first event with an energy of $107 \mathrm{TeV}$ was registered in 2015 (the distance to the cluster axis is 68 meters). The second event was registered in 2016 inside the geometric volume of the installation and has a reconstructed energy of $155 \mathrm{TeV}$. The coordinates of these events in the equatorial system are: right ascension $139.5^{\circ}$ and $173.4^{\circ}$, declination $5.6^{\circ}$ and $13.9^{\circ}$, respectively for the first and second candidate.

The most interesting source of variable luminosity for the recent research was the galaxy NGC 4993, where two neutron stars merged and produced a gravitational wave that 
was registered by the detectors LIGO and Virgo on August 17, 2017 (GW170817). The search for neutrino events in the direction from this source with the Baikal-GVD telescope was carried out using the cascade mode, with two different search intervals: $\pm 500 \mathrm{~s}$ around the gravitational wave event in the first case, and 14 days after the registration of the gravitational wave in the second case. The zenith angle NGC 4993 at the time of registration was $93.3^{\circ}$. No events from NGC 4993 in the specified time intervals were detected. From the absence of candidate events, upper limits on neutrino fluence have been derived [11].

\section{Conclusions}

The ultimate goal of the Baikal-GVD project is the construction of a $\mathrm{km}^{3}$-scale neutrino telescope comprising about ten thousand light sensors. Currently, the first three clusters of the telescope including 864 optical modules have been put into operation. A laser light source located in the central part of the three clusters ensures their precise joint calibration. The new time synchronization system provides an accuracy of combining event information from different clusters at the level of 3 ns. Additional studies of the acoustic positioning system of the telescope confirmed the value of the time uncertainty associated with the OM coordinate measurement on the level of $1 \mathrm{~ns}$.

The analysis of data collected in 2015-2017 allows for the selection of a sample of upward through-going muons representing candidate neutrino events and the identification of the first two promising high-energy cascade events - candidates for events from astrophysical neutrinos. The search for neutrinos associated with GW170817 with BaikalGVD allowed deriving upper limits on the neutrino spectral fluence from this source.

The commissioning of the first stage of the Baikal neutrino telescope, GVD-1, with an effective volume $0.4 \mathrm{~km}^{3}$ is envisaged for 2020-2021.

This work was supported by the Russian Foundation for Basic Research (Grants 16-2913032 and 17-02-01237).

\section{References}

[1] A. Avrorin et al., Nucl. Instr. and Meth. A639, 1 (2011)

[2] A. Avrorin et al., PoS (ICRC2017) 1034 (2017)

[3] A. Avrorin et al., EPJ Web Conf. 116, 01003 (2016)

[4] A. Avrorin et al., Instr. Exper. Tech. 57, 262 (2014)

[5] A. Avrorin et al., Nucl. Instr. and Meth. A742, 82 (2014)

[6] A. Avrorin et al., Physics of Particle and Nuclei 46, 211 (2015)

[7] A. Avrorin et al., these proceedings, talk presented by M.D. Shelepov

[8] A. Avrorin et al., these proceedings, talk presented by A.D. Avrorin

[9] A. Avrorin et al., // http://arxiv.org/abs/1808.10353

[10] A. Avrorin et al., these proceedings, talk presented by Zh.-A.M. Dzhilkibaev

[11] A. Avrorin et al., JETP Letters, 108, 12 (2018) 